\title{
LEVEL CROSSINGS OF A RANDOM TRIGONOMETRIC POLYNOMIAL
}

\author{
KAMBIZ FARAHMAND
}

(Communicated by William D. Sudderth)

\begin{abstract}
This paper provides an asymptotic estimate for the expected number of $K$-level crossings of the random trigonometric polynomial $g_{1} \cos x+$ $g_{2} \cos 2 x+\ldots+g_{n} \cos n x$, where $g_{j}(j=1,2, \ldots, n)$ are independent normally distributed random variables with mean $\mu$ and variance one. It is shown that the result for $K=\mu=0$ remains valid for any finite constant $\mu$ and any $K$ such that $\left(K^{2} / n\right) \rightarrow 0$ as $n \rightarrow \infty$.
\end{abstract}

\section{INTRODUCTION}

Let

$$
T(x) \equiv T_{n}(x, \omega)=\sum_{j=1}^{n} g_{j}(\omega) \cos j x
$$

where $g_{1}(\omega), g_{2}(\omega), \ldots, g_{n}(\omega)$ is a sequence of independent random variables defined on a probability space $(\Omega, \mathscr{A}, P)$, each normally distributed with finite mathematical expectation $\mu$ and variance one. The set of equations $y=T(x)$ represents a family of curves in the $x y$-plane. Some years ago Dunnage [2] showed that the number of times all save a certain exceptional set of these curves crosses the $x$-axis in the interval $0 \leq x \leq 2 \pi$ is $(2 n) / \sqrt{3}+O\left\{n^{11 / 13}(\log n)^{3 / 13}\right\}$. The measure of his exceptional set does not exceed $(\log n)^{-1}$. Later Sambandham and Renganathan [7] and Farahmand [3] considered the case when $\mu \neq 0$ and found that the expected number of crossings of this family with the $x$-axis is asymptotic to $2 n \sqrt{3}$. This asymptotic number of crossings remains invariant in the work of Farahmand [4] when, for $\mu=0$, he considered the crossings of this family with the line $y=K$ (i.e., with level $K)$, where $K$ is any real value constant such that $\left(K^{2} / n\right) \rightarrow 0$ as $n \rightarrow \infty$.

Here we consider the effect of $\mu$ being nonzero on the $K$-level crossings of this family of curves, and show even in this case the above asymptotic number

Received by the editors July 6, 1989 and, in revised form, September 19, 1989.

1980 Mathematics Subject Classification (1985 Revision). Primary 60G99; Secondary 42BXX.

Key words and phrases. Number of real roots, Kac-Rice formula, random trigonometric polynomial. 
of crossings persists. Denote by $N(\alpha, \beta) \equiv N_{K}(\alpha, \beta)$ the number of times that the family of curves $y=T(x)$ crosses the line $y=K$ on the interval $\alpha \leq x \leq \beta$, and let $E N(\alpha, \beta)$ be its expectation. We prove the following.

Theorem. If the coefficients of $T(x)$ in (1.1) are independent normally distributed random variables with mean $\mu$ and variance one, then for all sufficiently large $n$ and any constant $K$ the expected number of real roots of the equation $T(x)=K$ satisfies

$$
E N(0,2 \pi)=(2 n) / \sqrt{3}+O\left(n^{3 / 4}\right) \quad \text { if } K=O\left(n^{3 / 8}\right)
$$

and

$$
E N(0,2 \pi)=(2 n) / \sqrt{3}+o(n) \quad \text { if } K=o(\sqrt{n}) .
$$

2. A FORMULA FOR THE NUMBER OF CROSSINGS

Let

$$
\Phi(t)=(2 \pi)^{-1 / 2} \int_{-\infty}^{t} \exp \left(-y^{2} / 2\right) d y
$$

and

$$
\phi(t)=\Phi^{\prime}(t)=(2 \pi)^{-1 / 2} \exp \left(-t^{2} / 2\right),
$$

then by using the expected number of level crossings given by Cramer and Leadbetter [1, p. 285] for our equation $T(x)-K=0$ we can obtain

$$
E N(\alpha, \beta)=\int_{\alpha}^{\beta}(B / A)\left(1-\lambda^{2}\right)^{1 / 2} \phi(\xi / A)[2 \phi(\eta)+\eta\{2 \Phi(\eta)-1\}] d x
$$

where

$$
\begin{aligned}
A^{2} & =\operatorname{Var}\{T(x)-K\}, \quad B^{2}=\operatorname{Var}\left\{T^{\prime}(x)\right\}, \\
\lambda & =(A B)^{-1} \operatorname{cov}\left[\{T(x)-K\}, T^{\prime}(x)\right], \quad \xi=E\{T(x)-K\}, \\
\eta & =B^{-1}\left(1-\lambda^{2}\right)^{-1 / 2}(\gamma-B \lambda \xi / A) \quad \text { and } \quad \gamma=E\left\{T^{\prime}(x)\right\} .
\end{aligned}
$$

Since the coefficients of $T(x)$ are independent normal random variables with mean $\mu$ and variance one we can easily find that

$$
\begin{aligned}
\xi & =\mu \sum_{j=1}^{n} \cos j x-K=m_{1}-K, \text { say, } \\
\gamma & =-\mu \sum_{j=1}^{n} j \sin j x=-m_{2}, \text { say } \\
A^{2} & =\sum_{j=1}^{n} \cos ^{2} j x, \quad B^{2}=\sum_{j=1}^{n} j^{2} \sin ^{2} j x, \\
C & =\sum_{j=1}^{n} j \sin j x \cos j x, \quad \lambda=-C / A B, \\
\eta & =\left\{C\left(m_{1}-K\right)-A^{2} m_{2}\right\} / A \Delta \text { and } \Delta^{2}=A^{2} B^{2}-C^{2} .
\end{aligned}
$$


Hence from (2.1) and since $\Phi(t)=1 / 2+(\pi)^{-1 / 2} \operatorname{erf}(t / \sqrt{2})$, we have the extension of the Kac-Rice formula [5].

$$
\begin{aligned}
& E N(\alpha, \beta) \\
& \begin{aligned}
E & \int_{\alpha}^{\beta}\left(\Delta / \pi A^{2}\right) \exp \left[\left\{-B^{2}\left(m_{1}-K\right)^{2}-A^{2} m_{2}^{2}+2 m_{2} C\left(m_{1}-K\right)\right\} /\left(2 \Delta^{2}\right)\right] d x \\
& +\int_{\alpha}^{\beta}(\sqrt{2} / \pi) A^{-3}\left|C\left(m_{1}-K\right)-A^{2} m_{2}\right| \exp \left\{-\left(m_{1}-K\right)^{2} / 2 A^{2}\right\} \\
& \quad \times \operatorname{erf}\left\{\left|C\left(m_{1}-K\right)-A^{2} m_{2}\right| / \sqrt{2} A \Delta\right\} d x \\
\text { 2.2) } & \int_{\alpha}^{\beta} I_{1}(x) d x+\int_{\alpha}^{\beta} I_{2}(x) d x .
\end{aligned}
\end{aligned}
$$

3. OVERVIEW OF THE PROOF OF THE THEOREM AND SOME APPROXIMATIONS

In order to evaluate the expected number of real roots we divide the real roots into two groups: (i) those lying in the intervals $(0, \varepsilon),(\pi-\varepsilon, \pi+\varepsilon)$ and $(2 \pi-\varepsilon, 2 \pi)$ and (ii) those lying in the intervals $(\varepsilon, \pi-\varepsilon)$ and $(\pi+\varepsilon, 2 \pi-\varepsilon)$. For the roots (i) which, it so happens, are negligible, we need some modification to apply Dunnage's [2] approach, which is based on an application of Jensen's theorem [8, p. 125] or [6, p. 332]. For roots (ii) we use the Kac-Rice formula (2.2). The $\varepsilon$ should be chosen positive and small enough to facilitate handling type (i) roots, but large enough to allow for the approximation needed to discuss type (ii) roots. We choose $\varepsilon=n^{-1 / 4}$.

The function

$$
S(x)=\sin (2 n+1) x / \sin x
$$

and, defined by continuity at $x=j \pi$, will occur frequently and so it is convenient to collect together some related inequalities. Since for $\varepsilon \leq x \leq \pi-\varepsilon$ or $\pi+\varepsilon \leq x \leq 2 \pi-\varepsilon$ we have $|S(x)|<1 / \sin \varepsilon$, we can obtain

$$
S(x)=O(1 / \varepsilon) \text {. }
$$

Further

$$
S^{\prime}(x)=(2 n+1) \cos (2 n+1) x / \sin x-\cot x S(x)=O(n / \varepsilon)
$$

and

$$
\begin{aligned}
S^{\prime \prime}(x)= & -(2 n+1)^{2} S(x)-(2 n+1) \cos x \cos (2 n+1) x \sin ^{-2} x \\
& -\cot x S^{\prime}(x)+\operatorname{cosec}^{2} x S(x)=O\left(n^{2} / \varepsilon\right) .
\end{aligned}
$$

Hence from (3.1) and since by expanding $\sin x\left(1+2 \sum_{j=1}^{n} \cos 2 j x\right)$, we can show that

$$
\sum_{j=1}^{n} \cos 2 j x=(1 / 2)\{S(x)-1\}
$$


for this range of $x$ we have

$$
A^{2}=(1 / 2) \sum_{j=1}^{n}(1+\cos 2 j x)=n / 2+(1 / 4)\{S(x)-1\}=n / 2+O(1 / \varepsilon) .
$$

Also from (3.3) and since from (3.4)

$$
S^{\prime \prime}(x)=-8 \sum_{j=1}^{n} j^{2} \cos 2 j x=4 \sum_{j=1}^{n} j^{2}\left(2 \sin ^{2} j x-1\right)
$$

we have

$$
B^{2}=n(n+1)(2 n+1) / 12+(1 / 8) S^{\prime \prime}(x)=n^{3} / 6+O\left(n^{2} / \varepsilon\right) .
$$

From (3.2) we obtain

$$
C=-(1 / 2) \frac{d}{d x}\left(A^{2}\right)=-(1 / 8) S^{\prime}(x)=O(n / \varepsilon) .
$$

Also from (3.1), (3.2), and (3.4) we can easily obtain the two following estimates:

$$
m_{1}=(\mu / 2)\{S(x / 2)-1\}=O(1 / \varepsilon)
$$

and

$$
m_{2}=(-\mu / 4) S^{\prime}(x / 2)=O(n / \varepsilon) .
$$

Then (3.5), (3.6), and (3.7) give

$$
\Delta^{2}=n^{4} / 12+O\left(n^{3} / \varepsilon\right) .
$$

\section{PROOF OF THE THEOREM}

From (2.2) and (3.5)-(3.10) we can obtain

$$
\int_{\varepsilon}^{\pi-\varepsilon} I_{1}(x) d x=(n / \sqrt{3})\{1+O(\varepsilon)\} \exp \left\{-K^{2} / n+O\left(1 / n \varepsilon^{2}\right)\right\}
$$

and

$$
\int_{\varepsilon}^{\pi-\varepsilon} I_{2}(x) d x=O\left\{\left(n^{1 / 2} / \varepsilon\right) \exp \left(-1 / n \varepsilon^{2}-K^{2} / n\right)\right\}
$$

Hence from (2.2), (4.1), and (4.2) for $K=O\left(n^{3 / 8}\right)$, we have

$$
E N(\varepsilon, \pi-\varepsilon)=E N(\pi+\varepsilon, 2 \pi-\varepsilon)=n / \sqrt{3}+O\left(n^{3 / 4}\right)
$$

and for $K=o(\sqrt{n})$

$$
E N(\varepsilon, \pi-\varepsilon)=E N(\pi+\varepsilon, 2 \pi-\varepsilon)=n / \sqrt{3}+o(n) .
$$

Now we show that the expected number of real roots in the intervals $(0, \varepsilon)$, $(\pi-\varepsilon, \pi+\varepsilon)$, and $(2 \pi-\varepsilon, 2 \pi)$ is negligible. The period of $T(x)$ is $2 \pi$ and so the number of zeros in $(0, \varepsilon)$ and $(2 \pi-\varepsilon, 2 \pi)$ is the same as the number in $(-\varepsilon, \varepsilon)$. We shall therefore confine ourselves to this last interval; the interval 
$(\pi-\varepsilon, \pi+\varepsilon)$ can be treated in exactly the same way to give the same result. We consider the random integral function of the complex variable $z$,

$$
T(z, \omega)-K=\sum_{j=1}^{n} g_{i}(\omega) \cos j z-K .
$$

We are seeking an upper bound to the number of real roots in the segment of the real axis joining the points $\pm \varepsilon$, and this certainly does not exceed the number of real roots in the circle $|z|<\varepsilon$. Let $N(r) \equiv N(r, \omega, K)$ denote the number of real roots of $T(z, \omega)-K=0$ in $|z|<r$. By Jensen's theorem [8, p. 125] or $[6$, p. 332]

$$
\begin{aligned}
\int_{\varepsilon}^{2 \varepsilon} r^{-1} N(r) d r & \leq \int_{0}^{2 \varepsilon} r^{-1} N(r) d r \\
& =(2 \pi)^{-1} \int_{0}^{2 \pi} \log \left|\left[\left\{T\left(2 \varepsilon e^{i x}, \omega\right)-K\right\} /\{T(0)-K\}\right]\right| d x,
\end{aligned}
$$

assuming that $T(0) \neq K$, from which we have

$$
N(\varepsilon) \log 2 \leq(2 \pi)^{-1} \int_{0}^{2 \pi} \log \left|\left[\left\{T\left(2 \varepsilon e^{i x}, \omega\right)-K\right\} /\{T(0)-K\}\right]\right| d x .
$$

Now since the distribution function of $T(0, \omega)=\sum_{j=1}^{n} g_{j}(\omega)$ is

$$
G(x)=(2 \pi n)^{-1 / 2} \int_{-\infty}^{x} \exp \left\{-(t-\mu)^{2} / 2 n\right\} d t
$$

we can see that for any positive $\nu$

$$
\begin{aligned}
\operatorname{Prob}\left(-e^{-\nu} \leq T(0)-K<e^{-\nu}\right) & =(2 \pi n)^{-1 / 2} \int_{K-e^{-\nu}}^{K+e^{-\nu}} \exp \left\{-(t-\mu)^{2} / 2 n\right\} d t \\
& <(2 / n \pi)^{1 / 2} e^{-\nu} .
\end{aligned}
$$

Also we have

$$
\left|T\left(2 \varepsilon e^{i x}\right)\right|=\left|\sum_{j=1}^{n} g_{j} \cos \left(2 j \varepsilon e^{i x}\right)\right| \leq n e^{2 n \varepsilon} \max \left|g_{j}\right|
$$

where the maximum is taken over $1 \leq j \leq n$. The distribution function of $\left|g_{j}\right|$ is

$$
F(x)= \begin{cases}\sqrt{2} / \pi \int_{0}^{x} \exp \left\{-(t-\mu)^{2} / 2\right\} d t, & x \geq 0 \\ 0, & x<0\end{cases}
$$

and so for any positive $\nu$ and all sufficiently large $n$

$$
\begin{aligned}
\operatorname{Prob}\left\{\max \left|g_{j}\right|>n e^{\nu}\right\} & \leq n \operatorname{Prob}\left\{\left|g_{1}\right|>n e^{\nu}\right\} \\
& =n \sqrt{2 \pi} \int_{n e^{\nu}}^{\infty} \exp \left\{-(t-\mu)^{2} / 2\right\} d t \\
& \sim \sqrt{2 / \pi} \exp \left\{-\nu-\left(n e^{\nu}-\mu\right)^{2} / 2\right\} .
\end{aligned}
$$


Therefore from (4.7) and (4.8) except for sample functions in an $\omega$-set of measure not exceeding $(2 / \pi)^{1 / 2} \exp \left\{-\nu-\left(n e^{\nu}-\mu\right)^{2} / 2\right\}$,

$$
\left|T\left(2 \varepsilon e^{i x}\right)\right|<n^{2} \exp (2 n \varepsilon+\nu) .
$$

Hence from (4.6), (4.9) and since

$$
\left|n^{2} \exp (2 n \varepsilon+\nu)-K\right|<2 n^{2} \exp (2 n \varepsilon+\nu)
$$

if $K=o(\sqrt{n})$ or if $K=O\left(n^{3 / 8}\right)$, we obtain

$$
\begin{aligned}
\left|\left\{T\left(2 \varepsilon e^{i x}, \omega\right)-K\right\} /\{T(0, \omega)-K\}\right| & \leq e^{\nu}\left|2 n^{2} \exp (2 n \varepsilon+\nu)-K\right| \\
& \leq 2 n^{2} \exp (2 n \varepsilon+2 \nu)
\end{aligned}
$$

except for sample functions in an $\omega$-set of measure not exceeding

$$
(2 / n \pi)^{1 / 2} e^{-\nu}+(2 / \pi)^{1 / 2} \exp \left\{-\nu-\left(n e^{\nu}-\mu\right)^{2} / 2\right\} .
$$

Therefore from (4.5) and (4.10) we can show that outside the exceptional set

$$
N(\varepsilon) \leq(\log 2+2 \log n+2 n \varepsilon+2 \nu) / \log 2 .
$$

Because $\varepsilon=n^{-1 / 4}$, it follows from (4.11) and for all sufficiently large $n$ (4.12)

$$
\operatorname{Prob}\{N(\varepsilon)>3 n \varepsilon+2 \nu\} \leq(2 / n \pi)^{1 / 2} e^{-\nu}+(2 / \pi)^{1 / 2} \exp \left\{-\nu-\left(n e^{\nu}-\mu\right)^{2} / 2\right\} .
$$

Let $n^{\prime}=\left[3 n^{3 / 4}\right]$ be the greatest integer less than or equal to $3 n^{3 / 4}$; then from (4.12) and for $n$ large enough we obtain

$$
\begin{aligned}
E N(\varepsilon) & =\sum_{j>0} \operatorname{Prob}\{N(\varepsilon) \geq j\} \\
& =\sum_{1 \leq j \leq n^{\prime}} \operatorname{Prob}\{N(\varepsilon)>j\}+\sum_{j \geq 1} \operatorname{Prob}\left\{N(\varepsilon)>n^{\prime}+j\right\} \\
& \leq n^{\prime}+(2 / n \pi)^{1 / 2} \sum_{j \geq 1} e^{-j / 2}+(2 / \pi)^{1 / 2} \sum_{j \geq 1} \exp \left\{-j / 2-\left(n e^{j}-\mu\right)^{2} / 2\right\} \\
\text { (4.13) } \quad & =O\left(n^{3 / 4}\right) .
\end{aligned}
$$

Finally (4.3), (4.4), and (4.13) complete proof of the theorem.

\section{ACKNOWLEDGMENT}

The author wishes to thank the referee for his valuable comments.

\section{REFERENCES}

1. H. Cramer and M. R. Leadbetter, Stationary and related stochastic processes, Wiley, New York, 1967.

2. J. E. A. Dunnage, The number of real zeros of a random trigonometric polynomial, Proc. London Math. Soc. 16 (1966), 53-84. 
3. K. Farahmand, On the number of real zeros of a random trigonometric polynomial: coefficient with non-zero mean, Stochastic Anal. and Appl. 5 (1987), 379-386.

4. $\ldots$, On the average number of level crossings of a random trigonometric polynomial Annal. of Prob. 18 (1990).

5. S. O. Rice, Mathematical theory of random noise, Bell System Tech. J. 25 (1945), 46-156.

6. W. Rudin, Real and complex analysis, 2nd ed. McGraw-Hill, 1974.

7. M. Sambandham and N. Renganathan, On the number of real zeros of a random trigonometric polynomial: coefficient with non-zero means, J. Indian Math. Soc. 45 (1981), 193-203.

8. E. C. Titchmarsh, The theory of functions, 2nd ed. Oxford University Press, 1939.

Department of Mathematical Statistics, University of Cape Town, Rondebosch 7700, SOUTH AFRICA 